\title{
Capítulo 3 \\ El telón de fondo del discurso estudiantil: entre la tecnología como arma de doble filo y un mundo digitalmente reconstruido
}

continuación, se presentan algunos hallazgos que permiten
comprender las coordenadas en las que se inscribe el discurso estudiantil sobre el papel de las tecnologías en el mundo actual. En primer lugar, se intentó recomponer el entramado de significados que los participantes asociaron a la tecnología en general y a las tecnologías digitales en particular. Según ellos, la tecnología opera en la sociedad de modo ambivalente, generando situaciones tanto positivas como negativas. De ahí que se la defina como arma de doble filo. Sin embargo, esas valoraciones ambivalentes no se restringen a la tecnología, sino que también se aprecian en sus descripciones sobre el mundo actual. La globalización, el acceso a la información y la comunicación digitalmente mediada, además de ser problemáticas típicas del tiempo presente, involucran una serie de riesgos y oportunidades tanto para el individuo como para la sociedad en general. 


\section{La tecnología, un arma de doble filo que cambia el mundo}

En principio, el objetivo de iniciar las discusiones hablando de la tecnología en general fue sacar a la luz las definiciones e imaginarios más gruesos asociados a este concepto. Sin embargo, a medida que la discusión avanzó, los participantes empezaron a hacer referencia a tecnologías específicas, como ordenadores, celulares, Internet, etc., que funcionaron como "activadores culturales" (Jenkins, 2008), o sea, como catalizadores de significados compartidos. Este giro respecto del objetivo trazado inicialmente resultó bastante provechoso, pues permitió integrar experiencias y anécdotas más individuales al hilo del debate.

Una de las primeras cosas que hay que mencionar fue que los estudiantes tendieron a definir y valorar las tecnologías de modo oposicional, es decir, en términos de bueno vs. malo, de provechoso vs. peligroso, o de positivo vs. negativo, etc. Hubo, incluso, algunos participantes que se refirieron enfáticamente a la tecnología como "arma de doble filo".

La relación entre tecnologías y cambio social también fue otro asunto sobre el que los estudiantes hicieron hincapié. Inclusive, los participantes demostraron mucho más interés en discutir sobre las dimensiones comunicativas, culturales y socioeconómicas de los cambios generados por la tecnología que sobre las tecnologías en sí mismas. Intervenciones como las que siguen ilustran lo dicho hasta aquí:

-El mundo ha cambiado. [La tecnología] hace unir a las personas, que se puedan comunicar, y se logra facilitar la comunicación. No se puede llevar una vida dependiente de eso, pero uno tiene que cumplir con unas cosas para sobrevivir (...). Es como una herramienta, sí, pero una herramienta de doble filo. En el presente a mí me puede ayudar hartísimo, pero de pronto en el futuro... (G. 1, p. 14, r. 1) 
-Con respecto al otro tema [las tecnologías]: nos alejan un poco de las personas que están cerca y nos acercan a los que están lejos: son como un arma de doble filo. (G.10, p.1, r.7)

-Considero que esto [tecnología] es un arma de doble filo. Parto de mi experiencia: yo no soy de acá y aunque no tengo familia en otro país, estando en el mismo país puedo sentirlos cerca (...) por un momento con un clic verles la cara (...) reconforta el corazón y las distancias se acortan por minutos; pero pues no es lo mismo ver a una persona en una pantalla a verla, a sentirla directamente (...) digo que es un arma de doble filo porque (...) veo que se crean vicios: la gente empieza a aparentar lo que no es, a mostrar una faceta diferente a lo que es. Y no solo se niegan a lo que son, sino que cortan con la posibilidad de darse a conocer al mundo. (G. 10, p. 4, r. 6)

No obstante, la metáfora del arma de doble filo enmarca un discurso que también vale la pena desmenuzar. En la Figura 1, se presentan algunos hallazgos más detallados. Como se puede observar, hay tres cualidades que definen, en el nivel más general, a las tecnologías: el avance, la eficiencia y la facilidad. Estas propiedades remiten, a su vez, a otras mucho más específicas, vinculadas sobre todo a las tecnologías digitales: la novedad, la portabilidad y la conectividad.

Conforme avanzaban las discusiones, los aportes de los estudiantes dejaron de restringirse a las propiedades intrínsecas de la tecnología y dieron paso a un intercambio de opiniones, experiencias y argumentos sobre su rol y su impacto en el mundo actual. Esto explica por qué en la Figura 1 la tecnología aparece asociada a cuestiones de índole sociocultural, como el prestigio y la exclusión.

A fin de comprender de modo más detallado las valoraciones de los estudiantes en torno a las tecnologías y el mundo actual, a continuación, se presentan los hilos más interesantes de la discusión. El contraste y, a la vez, el vínculo entre la autonomía y la dependencia, así como el tránsito de la facilidad a la pereza y del prestigio a la exclusión, constituyen muestras de que, para los 


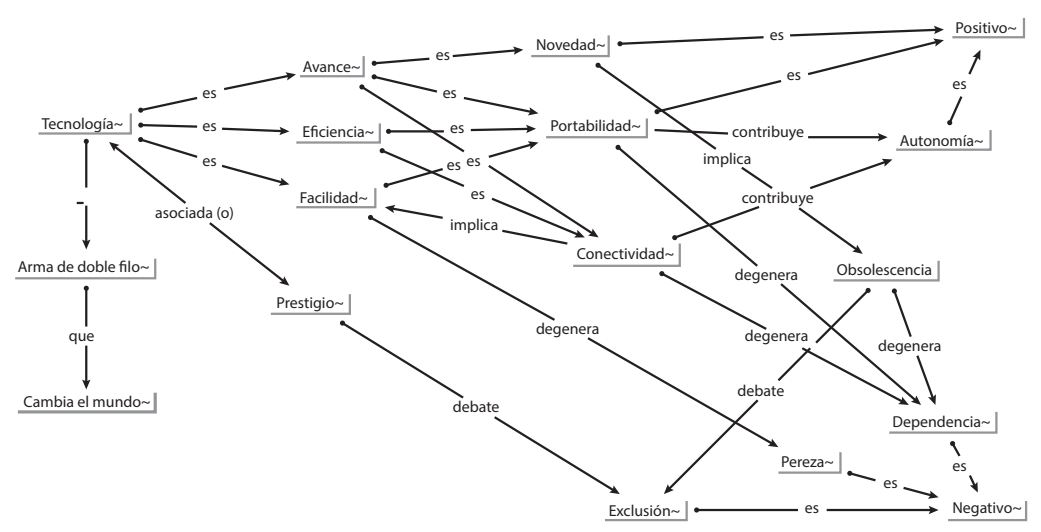

Figura 1. Propiedades asociadas a la tecnología.

Fuente: elaboración propia.

participantes, la tecnología es ambivalente y está asociada a cambios bastante significativos.

Entre la autonomía y la dependencia

En principio, en muchas definiciones de la tecnología se mencionó la palabra "avance", en alusión a un cierto poder de las tecnologías de mejorar la calidad de vida de las personas. Además, dijeron que el avance también tenía que ver con innovaciones a nivel de prestaciones y servicios que terminaban por reemplazar tecnologías más antiguas. Por ejemplo:

-Digamos que la asocio [la tecnología] más a todos esos avances de dispositivos que van dirigidos a mejorar la calidad de vida de las personas. (Grupo 3, pregunta 1, respuesta 2)

-[La tecnología] es una serie de avances de los que podemos echar mano para obtener mejor información; avances de aparatos electrónicos que cada vez el hombre actualiza y aprovecha. 
Entonces, sacan primero una cosa y luego más adelante algo parecido pero con más aplicaciones. (G. 2, p. 1, r. 10)

A la par de las menciones explícitas a los avances asociados a la tecnología, constantemente, se hacía alusión tácita a la eficiencia como segunda cualidad relevante. La resolución de problemas y el mejoramiento en la calidad de vida no dependen solamente de los avances tecnológicos, sino también del hecho de que dichos avances "optimizan", es decir, hacen más eficiente la ejecución de tareas y actividades.

-Yo trabajo con una compañía que vende tecnología. Digamos, los computadores, los servidores, todo ese tipo de tecnología hardware y software ayudan a que las empresas obviamente utilicen mejor esos recursos tecnológicos para optimizar los recursos que ellos manejan. (G. 3, p. 1, r. 1)

-Hay ámbitos que nosotros de pronto no exploramos mucho pero que son significativos y aportan mucho al mismo desarrollo de la sociedad. Creo que todo esto tiene un fin (...) mejorar la calidad de vida de las personas y optimizar en las empresas recursos como el tiempo y el movimiento. Para mí es eso (G. 3 , p. 1, r. 11).

El código "novedad", por su parte, recoge una serie de afirmaciones fuertemente vinculadas a la tecnología como avance, pero que se inscriben en un ámbito más próximo a la vida del estudiante, en el que hay mayor presencia de las tecnologías digitales y estas se perciben en la medida en que causan sorpresa. En este sentido, dos de ellos afirmaron:

-Lo que a uno más le llama la atención es lo último que sale. Las tecnologías antiguas igual uno tiende a olvidarlas. (G. 1, p. 10, r. 2)

-Yo considero que lo que más llama la atención, tanto a jóvenes como adultos, es la innovación del producto que está saliendo. (G. 1, p. 8, r. 3) 
La portabilidad y la conectividad también aparecieron como cualidades específicas de las tecnologías digitales, y su relación con la facilidad, el avance y la eficiencia se advierte en las siguientes intervenciones. En la primera, una estudiante de Comunicación responde a la pregunta por aquello que hace a ciertas tecnologías especialmente populares y atractivas. Luego, un estudiante de Ingeniería se refiere así a la importancia de la conectividad en el mundo actual:

-(...) que los aparatos sean más que todo portátiles, que pueda llevarlos. Un computador de mesa ya no tiene la misma atracción que tiene un portátil. Por la facilidad me llevo el portátil a la universidad y termino el trabajo allá, lo que no se podía hacer con un computador de mesa; es como la facilidad de las cosas. (G. 1, p. 2, r. 5)

-Las tablet, los smartphones y los portátiles los asocio, personalmente, porque cada vez la era de la información va avanzando más. Lo asocio porque todo el mundo ya trata de estar comunicado con todo el mundo, conectado con todas las personas. Y pues la era de la información está directamente relacionada con estos aparatos electrónicos porque ya tener la información en el bolsillo aporta mucho, no solo a nivel personal, sino profesional y ejecutivo. (G. 3, p. 1, r. 9)

Como se muestra en la Figura 1, la portabilidad y la conectividad constituyen uno de los puntos en los que el discurso empieza a bifurcarse entre lo positivo y lo negativo de las tecnologías (en especial las digitales). Por un lado, algunos afirmaron que la portabilidad y la conectividad dotan al individuo de mayor autonomía, sobre todo en lo que atañe al acceso a la información y al aprendizaje. Esto se evidencia en la siguiente intervención de una estudiante de Derecho:

-Me parece positivo, porque si lo planteamos a nivel de la construcción de los jóvenes y su aporte al conocimiento, podemos ver eso mismo que, digamos, antes estaban completamente 
controlados los parámetros de la educación. El Internet o estos aparatos tecnológicos lo que hacen es abrir puertas a que esas personas puedan ser leídas, consideradas, ya que pues en un nivel académico está muy restringido; eso hay una hegemonía fuerte de las personas. (G. 10, p. 3, r. 6)

Por otra parte, se argumentó que, al facilitar el acceso a la información y el contacto con las personas, la portabilidad y la conectividad pueden convertirse en motivos para que el hombre cree dependencia de las tecnologías digitales. Esta fue, de hecho, una de las cuestiones sobre las que se hizo más referencia en los grupos de discusión donde se abordaron estos temas. Cuando los participantes se referían a la dependencia de aparatos tecnológicos solían hacerlo desde sus experiencias personales, como si fuera un asunto que les resultase especialmente próximo.

- Nosotros somos muy dependientes de nuestros celulares. Yo creo que si a uno se le queda el celular en la casa ¡mejor dicho!, eso fue el caos total. (G. 3, p. 2, r. 9)

-(...) apenas prendes el computador las ganas te superan, no puedes tener esa fuerza de voluntad, sino que ya abres el Face y te quedas ahí. (G. 6, p. 4, r. 6)

-El Facebook es como una adicción, una necesidad para satisfacer $(. .$.$) hace parte de ti y te hace sentir parte de algo, de la$ sociedad. (G. 6., p. 8, r. 10)

La dependencia como situación negativa relacionada con las tecnologías digitales también tiene que ver, según los estudiantes, con unos ciclos cada vez más acelerados de relevo y obsolescencia tecnológica. Esto, apuntaron, hace que las tecnologías digitales degeneren en meros objetos de consumo. En palabras de una estudiante de Administración, "ese tipo de capitalismo y globalización hace como que todos estemos en el mismo punto, todos tenemos que estar con las mismas tecnologías para avanzar en este mundo globalizado" (G. 3, p. 3, r. 4). 
La dependencia, entonces, se entiende como una necesidad poderosa pero artificial - por ser socialmente construida- de adquirir y usar la versión más reciente de tal o cual dispositivo a fin de no quedar rezagado. Dicha relación entre avance y dependencia se ilustra en observaciones como estas:

-Hace diez años no sabíamos utilizarlas (tablets y smartphones) pero digamos que nos toca adaptarnos, (aunque) la mayoría se queda rezagada y tiene que acelerar los pasos para alcanzar. (G. 3, p. 5, r. 2)

-Si usted no vive usando tecnología usted está en la prehistoria. (G. 4, p. 11, r. 2)

De la facilidad a la pereza

El "doble filo" de las tecnologías también se advirtió en las discusiones sobre la facilidad (en tanto cualidad positiva de la tecnología) y la pereza (entendida como actitud individual típica del universo juvenil). La facilidad que brindan las tecnologías tiene que ver para los participantes con la capacidad de estas para hacer más cómoda la ejecución de ciertas tareas y actividades cotidianas, e incluso de ya $n o^{11}$ hacerlas más. En este sentido, la facilidad implica la posibilidad de que la tecnología suceda o remplace al humano en ciertos trabajos o tareas:

-[Las tecnologías] son herramientas que nos pueden facilitar de una y otra forma lo que hacemos, la vida misma. (G. 1, p.7, r. 3)

-Esas cosas inteligentes que se ven ahora (...) ya hacen todo, (...) ya no tendría que arreglar el cuarto porque lo hacen ahora por mí. Ya no tengo que hacer algo que no me gusta. (G. 2, p. 4, r. 3)

11 Es una expresión que sugiere un cambio significativo. Es recurrente en las intervenciones sobre este tópico, como se podrá constatar más adelante. 
$\mathrm{Al}$ pedirles más detalles sobre qué actividades y tareas concretas eran las que facilitaban las tecnologías, muchos estudiantes respondieron que la facilidad tenía que ver, principalmente, con tener acceso a recursos comunicativos e informacionales con menos esfuerzos y/o desplazamientos. Destacan las siguientes intervenciones, en las que se celebran las facilidades, entendidas como minimización del esfuerzo, que ofrece Internet en términos de comunicación y acceso a información:

-Uno ya no tiene que ir a la biblioteca, sino que en Internet ya lo encuentra todo. Anteriormente, uno recurría a los libros, pero ahora no; ahora se recurre al computador. (G. 2, p. 1, r. 5)

-A mí la biblioteca me queda como a unas siete cuadras y aun así antes me daba pereza sacar libros, entonces yo los compraba por Internet. Ahí si leo más porque los tengo como más al alcance. (G. 2, p. 5, r. 3)

-Mejora la comunicación. Digamos, yo la necesito a ella y yo le escribo y ya, sin necesidad de verme con ella y buscarla y llamarla ni nada. Es más fácil. (G. 2, p. 3, r. 3)

Conforme se abordaba el asunto de la facilidad, aparecieron algunas afirmaciones más críticas y pesimistas. Los estudiantes enfatizaron bastante en que la facilidad que ofrecen las tecnologías puede hacer que las personas se tornen perezosas y sedentarias. Una vez más, se advirtió cómo para los participantes una cualidad intrínseca de la tecnología puede devenir en amenaza al promover actitudes individuales nocivas (que incluso alguno de los estudiantes definió como facilismo).

-Sí, [la tecnología] ayuda a la persona (...) pero al mismo tiempo nos vuelve perezosos la facilidad de lo rápido. No vamos más allá, sino solo copiar y pegar. (G.8, p. 4, r. 4)

-A veces yo salía de paseo o a comprar ropa a un centro comercial, pero ahora entro a Mercado Libre. Me compro la que 
me gusta y ellos me lo traen, entonces es como muy facilista. (G. 2, p. 5, r. 13)

-Uno se vuelve sedentario, ya ni camina, se vuelve más gordo porque uno no tiene costumbres físicas por la tecnología. (G. 1, p. 17, r. 6$)$

\section{Del prestigio a la exclusión}

No todo el relato estudiantil sobre la tecnología se tejió alrededor de sus propiedades materiales. La relación que establecieron los participantes entre prestigio y exclusión merece, en este sentido, una mención especial, pues se puso de manifiesto que las tecnologías, en especial las digitales, eran símbolos para ellos con un considerable poder de evocación, sobre todo en sus relaciones entre pares.

El código "prestigio" se creó a partir de una discusión que tuvo lugar en el grupo 2, entre estudiantes de Comunicación y Sociología. Según algunos de ellos, el prestigio es un tipo de reconocimiento social asociado a la "capacidad de adquirir y renovar" (G. 2, p. 3, r. 20) tecnologías exclusivas, de gama alta y de última generación. El prestigio vendría a ser una ganancia simbólica asociada a dos fuerzas materiales: la tecnología (en particular los teléfonos móviles) y el dinero.

La discusión inició cuando una estudiante de Comunicación Social planteó la relación entre tecnología, poder adquisitivo y prestigio, en estos términos:

-Lo del prestigio es entonces el que más dinero tenga, porque si tiene la posibilidad de tener un celular que salió esta semana y luego sale otro la otra semana y tienes la posibilidad de tenerlo, eso te da también prestigio. Es la capacidad de adquisición que tengas (...). Dentro de los jóvenes es muy común. Tener un celular así, el último, te da cierto prestigio. Tú ves que hoy en día, por ejemplo, en Trasmilenio, mucha gente anda con la 
tablet. Entonces, está uno ahí mirando qué hacen. A eso me refiero, da como cierto “iay, tan chévere!". (G. 2, p. 3, r. 15 y 19)

La opinión de la estudiante dio lugar a una serie de reacciones que dejaron ver, además, cómo algunas marcas y productos específicos (iPhone, por ejemplo) destacaban en la cadena de relaciones que se arropaban bajo el concepto de prestigio. Las intervenciones de dos estudiantes de Comunicación Social e Ingeniería, respectivamente, dan cuenta de ese vínculo entre determinados teléfonos y lo que, para ellos, significa poseerlos:

-Yo digo que solo los que tienen un iPhone son los que tienen más posibilidades [económicas]. Sí, entonces yo lo veo de esa manera, yo creo que cualquier persona sabe que ese celular es muy caro, ejemplo un millón algo, y que si lo compra es porque tiene una situación económica buena para poder tenerlo (G. 2, p. 3, r. 18).

-(...) la tecnología se hace popular por la necesidad social del ser humano de reconocimiento, de aceptación. De una u otra forma tener un teléfono inteligente genera un reconocimiento social diferente a si no lo tiene. Yo, por ejemplo, tengo un iPhone y, digamos, socialmente me ven de forma diferente. Ocurre eso en la sociedad y no solo con las tecnologías de la comunicación sino con las tecnologías en general. Si tú tienes las tecnologías de punta eso te da un estatus. Además, está el tema social; necesitas estar conectado con la gente, necesitas ser reconocido. (G. 3, p. 3, r. 6)

La referencia al prestigio asociado a los teléfonos móviles en el ámbito juvenil dio lugar a algunas controversias bastante significativas, en la que quedó de manifiesto que las valoraciones sobre las tecnologías digitales no son solo de tipo racional sino también moral, estético y emocional. Todo ello se ilustra en el siguiente diálogo, en el que un grupo de estudiantes discute sobre lo que implica tener o no tener ciertos tipos de teléfonos móviles. 
- Lo que decía ella, todo el mundo con iPhone, y uno con celular ahí todo feíto. Hasta da pena sacarlo. ¿Que lo llamen a uno y uno en público? ¡Uy, no!

- Hay gente así, pero eso depende de la gente, de los valores de una persona, porque si es una persona que le importa cinco...

- Hay que decir la verdad. En nosotros se ve eso: todo el mundo aquí tiene iPhone, y que a uno lo vayan a llamar de un celular así (feo), a uno le da pena.

-Pues yo digo que no.

-Puede ser que sí, pero existen personas que no.

- Pues a mí no me daría pena. Yo lo tengo (iPhone) es porque me parece chévere chatear desde ahí y no tener que llamar (...).

-Ella también tiene un iPhone, pero entonces el mío es más feo que el de ella y entonces pues uno dice: "no, el de ella tiene más aplicaciones que el mío". Y eso no es tan chévere. (G. 2, p. 2, r. 25, 27-31)

Conforme se profundizaba en la cuestión del prestigio vinculado a ciertos aparatos tecnológicos, se empezó a debatir sobre sus implicaciones sociales negativas. Se empezó a hablar explícitamente de exclusión, entendida como el resultado de una clasificación social en función del acceso y del uso de ciertas tecnologías y marcas. Tres estudiantes declararon lo siguiente:

-Ahora clasificamos a las personas por eso del prestigio. (G. 2, p. 5, r. 2)

-Desafortunadamente las personas asocian la tecnología al estatus social, porque si tengo tecnología de punta soy más avanzado. (G. 3, p.7, r. 2)

-La adquisición [de tecnologías] aumenta el ego, esto implica que no evidenciamos ni reconocemos que hay personas que no 
tienen recursos suficientes ni los medios socioeconómicos $(. .$. y como no los tienen los ponemos a un lado. (G. 11, p. 1, r. 1)

Los estudiantes manifestaron, además, que la exclusión ocurre tanto en el nivel micro de la interacción entre jóvenes como en el nivel macro de las relaciones entre países. Sobre este asunto una estudiante de Negocios Internacionales afirmó:

-La comunicación también se ha hecho más rápida con los parientes y eso, pero también se ha hecho más excluyente. En los países de Tercer Mundo no tienen la capacidad de adquirir todas las tecnologías de última generación, como sí se puede hacer en Estados Unidos. Entonces, las personas que no tienen la capacidad de adquirir las tecnologías empiezan a ser vistas como marginados, como pobres. Yo creo que lo de la marginalidad es desde siempre, solo que antes era con otras cosas. Hoy, culturalmente, se ve a la persona que tiene celular como ¡wow! (G. 2, p. 5, r. 4)

La intervención de un estudiante de Derecho, en una de las últimas sesiones de trabajo, recoge bastante bien las conclusiones a las que, en general, llegaron los jóvenes tras debatir sobre la relación entre tecnología, prestigio y exclusión. Según él, la tecnología es un mal necesario para mantenerse vinculado a la sociedad actual y no resultar excluido.

-Desde mi punto de vista, podríamos ver que el uso de estas tecnologías se considera un mal necesario, ya que mediante la globalización estos elementos tecnológicos se vuelven una necesidad y las personas necesitan estos elementos para poder vincularse a la sociedad. (...) si tú no estás conectado mediante estos elementos (teléfonos móviles, tabletas, Internet) no tienes una inclusión social (...) [se produce] una marginación de las personas que no emplean los aparatos y las personas que no tienen los elementos ni las capacidades para poder tener control sobre estos; se genera una distinción y marginación de estas personas en la sociedad. (G. 10, p. 1, r. 5) 


\section{Mundo actual}

Conforme se fueron desarrollando las discusiones con los estudiantes, se advirtió que en sus intervenciones la tecnología no aparecía sola, sino aparejada a reflexiones sobre las relaciones sociales, la individualidad, la cultura y la economía, etc. Surgió, cada vez más frecuentemente, una serie de imaginarios sobre la realidad social que los estudiantes asociaban a sus experiencias con la tecnología. Por este motivo, se intentó reconstruir dichos imaginarios, a fin de comprender mejor, desde la perspectiva de los participantes, cómo es que la tecnología cambia el mundo y por qué se la describe como un arma de doble filo.

En la Figura 2, se resumen los hallazgos más significativos sobre la participación de las tecnologías en la configuración del mundo actual. En términos generales, pareciera que las tecnologías digitales introducen una forma particular de estar en el mundo, esto es, unos modos específicos de comunicación, de cooperación, de aprendizaje y de construcción identitaria. Dada la complejidad de estos asuntos, se organizaron los contenidos de las discusiones en dos niveles. El primero, está integrado por tres temas que, de acuerdo con lo que manifestaron los estudiantes, resultaron estructurales: (I) la comunicación mediada digitalmente, (II) la globalización y (III) los cambios en el acceso y la difusión de la información. El segundo nivel está compuesto por otras tres cuestiones igualmente significativas, pero que resultan de las interrelaciones entre dichos temas estructurales: (i) la construcción del conocimiento, (ii) las formas contemporáneas de cooperación y solidaridad y (iii) la construcción de la identidad.

Información, comunicación, y globalización: las grandes cuestiones del mundo actual

Para los estudiantes, la información es un recurso tan abundante como esencial. La facilidad en el acceso y el incremento de los 


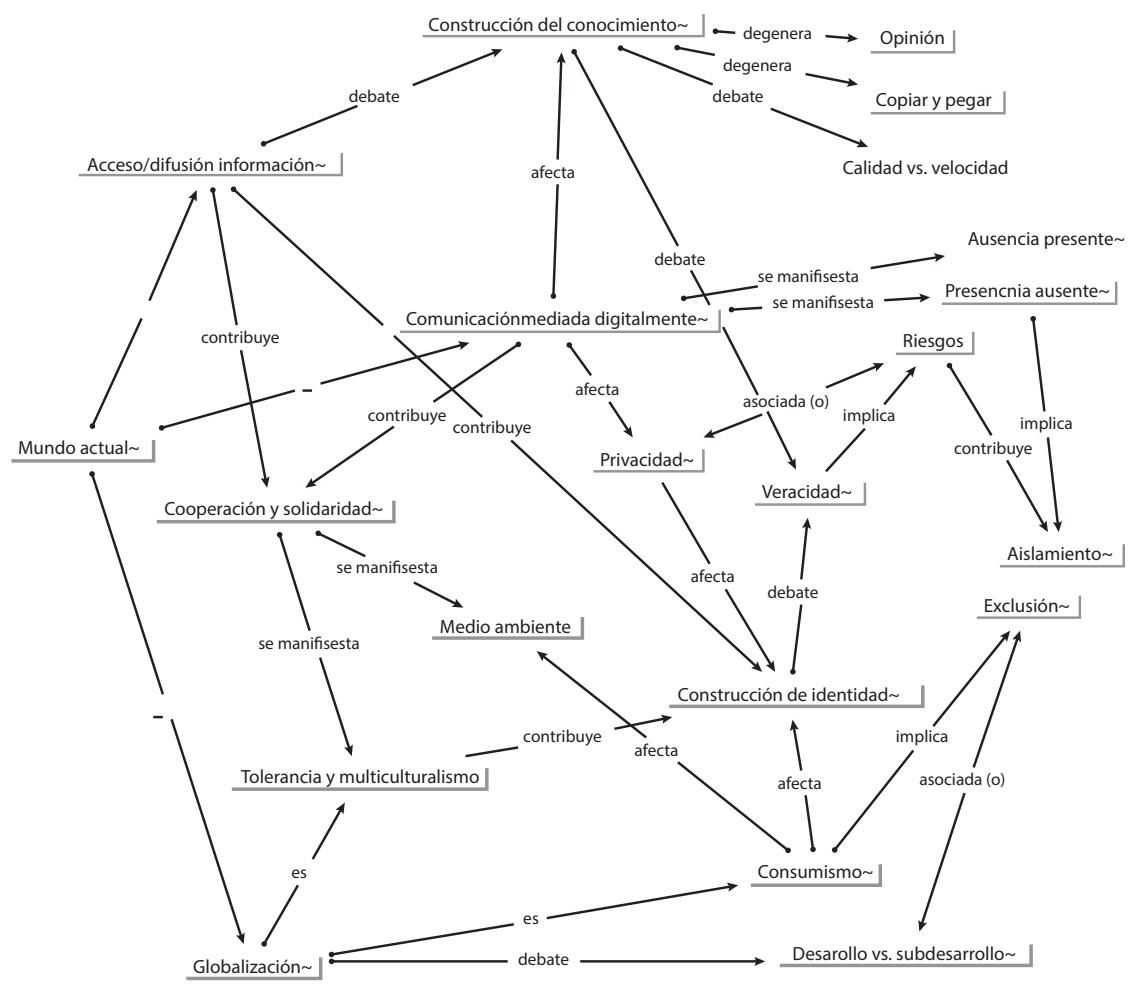

Figura 2. Mundo actual.

Fuente: elaboración propia.

medios de difusión de la información fueron asuntos a los que se hizo referencia insistentemente. Hubo un amplio consenso en torno a la idea de que el acceso a la información caracteriza el mundo actual y lo diferencia respecto del pasado. Para la mayoría de los participantes, estos cambios son bastante positivos, pues ensanchan el horizonte de posibilidades en lo que se refiere al aprendizaje, el trabajo y el entretenimiento. Esto se advierte en las siguientes afirmaciones:

-El mundo actual es mejor en lo que tiene que ver con buscar información. 
-[Ahora] la gente vive más informada y con más conocimiento. La otra vez yo estaba viendo un documental y mi abuelo se sentó conmigo y empezó a verlo. Y entonces me cuenta que hay cosas de antes que no se sabían, que ese tipo de historias no se contaban en el colegio. En cambio hoy en día uno entra a Internet y busca sobre historia, por ejemplo, y hay tantas cosas: hay textos y videos. Entonces uno ahora se puede informar más. (G 2, p. 5., r 2 y 14)

-Para mí, es vital estar conectado porque lastimosamente aquí en Colombia no hay mucha información en físico de mi estilo de música, de lo que yo hago, de lo que me gusta aprender; y pues para pagar un profesor no tengo los recursos ni el tiempo [entonces] todo lo hago desde mi casa. (G. 6, p. 2, r. 7)

Según buena parte de los estudiantes, Internet es un recurso fundamental en el desarrollo de su personalidad, pues permite superar barreras culturales de índole local al darles acceso a información y recursos que resultan esenciales para su formación identitaria. En este nivel destacan los siguientes aportes:

-En el pasado, una persona que no tenía la información -voy a poner un ejemplo de lo que son los LGBTI-, de pronto antes a la gente le daba miedo decir: "yo soy esto y tal", porque pues no hay nadie más como yo. Ahora ellos pueden buscar y ver que existen comunidades que se encargan de ayudarlos, de decirles que no que no está tan malo, que hay personas iguales a ellos. Eso también ayuda, entonces yo no soy un extraño ni nada, también tengo personas que son iguales a mí. (G. 2, p. 7, r. 2)

-(...) ya a uno como que le toca tener la mente un poquito más abierta; la gente no es solo la que está aquí, sino hay muchos otros miles de personas que tienen otras costumbres. Yo me la paso mucho en Tumblr y más que todo son gente de Estados Unidos. La gente que publica las cosas, todo lo que hacen, uno ve partes de una cultura diferente, uno ve que no es solo lo que uno cree. En mi casa, por ejemplo, no estaba bien visto que yo fuera vegetariana, pero en otros lugares se ve superbien eso. Entonces, si yo creí que eso (comer carne) era malo, algo que 
no se veía bien al igual que eso del LGBTI, es de respeto y de tolerancia. (G. 2, p. 7, r. 3)

Ahora bien, si por un lado la información es para los estudiantes un recurso básico y valioso, por otro la comunicación mediada por dispositivos y plataformas digitales aparece como una cuestión multidimensional, transversal y polémica. Según ellos, la comunicación mediada digitalmente tiene que ver, sobre todo, con unos límites difusos entre ausencia y presencia, con una mutación profunda en la noción de la privacidad, y con cambios negativos en el estilo de vida, tales como el aislamiento y el sedentarismo.

Las discusiones con los estudiantes dejaron entrever que, para ellos, por medio de la comunicación se crean los vínculos sociales y se los dota de sentido. Según afirmaron, dado que la comunicación actual está mediada digitalmente, el vínculo social termina siendo definido, de forma problemática y ambivalente, por las posibilidades y los límites que imponen ciertos dispositivos. Por esta razón, si bien los participantes celebran la eficiencia que introducen las tecnologías digitales en los procesos comunicativos, pues "hacen unir a las personas y se logra facilitar la comunicación” (G. 1, p. 13, r. 1), también se quejan de que estas generan pérdida de cercanía, de empatía y de contacto con los otros. En sus propias palabras:

-Ya no se ve tanto la comunicación como de contacto personal. Yo ya, por eso, ni le oigo la voz a la persona, ni sé cómo se expresa porque como estoy ahí chateando (...). Digamos que el mundo ha cambiado porque se ha llenado como de esa tecnología, todo el mundo chatea, entonces, todo el mundo va por ahí metido en sus cosas; entonces, se ha perdido como la comunicación verbal que se tenía. (G. 2, p. 5, r. 1)

Cuando se pidió a los estudiantes referirse a la comunicación en el mundo actual, muchos afirmaron que la mediación de las tecnologías digitales amenaza las convenciones tradicionales sobre la comunicación humana, sobre todo en lo que respecta a la 
importancia del contacto personal y del encuentro local. Sin embargo afirmaron, la comunicación mediada digitalmente privilegia y promueve contactos virtuales en escenarios no locales, como los que acontecen en las redes sociales.

A juicio de los participantes, los cambios en la esfera de la comunicación generan dos tipos de actitudes, que constituyen la manifestación más clara y recurrente de este nuevo orden comunicacional: la ausencia presente y la presencia ausente ${ }^{12}$. Para la muestra, la discusión que sigue:

-Me parece que en la parte comunicativa [las tecnologías digitales] fortalecen las [relaciones] no locales (...) y debilitan mucho las locales, pero por eso mismo: por el espacio y el tiempo que se le da a esas (relaciones sociales) no locales: por chats, por redes, por muchas cosas se le da mucho tiempo a eso y se descuidan las locales.

-(...) estamos en una era en que nos importa mucho más tener un contacto no físico, más escribir que estar mirando una persona o algo. (...) se trata de eso, de establecer una relación completa con una persona que no está acá (...).

-Mi opinión sería como que debilita las [relaciones sociales] locales, al punto que uno pasa más tiempo con las personas que no están a su alrededor.

-Las relaciones locales sí se debilitan mucho debido a que todo el mundo anda en las mismas; es más fácil responder por el celular que ponerle cuidado a la persona que se tiene al lado. $(\mathrm{G}$ 9 , p. 3, r. 2-5)

12 Ambos tropos, presentes en el trabajo de Lee y Wellman (2012), remiten al hecho de que las tecnologías digitales permiten a los individuos comunicarse con quienes están físicamente lejos (ausencia presente), al tiempo que los sustraen de las conversaciones e interacciones que tienen lugar en su entorno físico más inmediato (presencia ausente). 
Dado que la comunicación mediada digitalmente privilegia lo virtual por sobre lo local y lo presencial, el aislamiento y el sedentarismo se advierten como riesgos a nivel social, físico y psicológico. Algunos participantes se refirieron al aislamiento como el resultado de una dependencia franca de las tecnologías digitales. Ello se manifiesta en una actitud permanente de presencia ausente que termina por erosionar la comunicación con quienes están físicamente cerca. Según ellos, en el mundo actual las personas "se la pasan pegadas a un computador y ya no tienen vida social" (G. 2, p. 5, r. 9), por lo que "se pierde el sentido colectivo y se vuelven más individualistas" (G. 10, p. 1, r. 3). Inclusive, el aislamiento, en tanto opción individual, adquiere tintes dramáticos en afirmaciones como esta, de una estudiante de Derecho: "dejamos de lado las personas que nos importan, con quienes compartimos tiempo (...) nos estamos perdiendo lo mejor de la vida (...) estamos con una persona físicamente, pero no estamos con ella en realidad" (G. 10, p. 8, r. 8).

Para los participantes, la comunicación mediada digitalmente también redefine el ámbito y el significado de lo privado, de lo íntimo. Si bien sus intervenciones evidenciaban que para ellos la privacidad era un asunto relevante, parecía que lo que más les preocupaba era el grado de control sobre la información que circula por las redes sociales. En otras palabras, los cambios asociados a la privacidad, per se, no parecían estar en el centro de sus preocupaciones, sino más bien el riesgo que supone quedar expuestos. Lo que realmente parecía inquietarles era, entonces, que su imagen se viera afectada, y no tanto que los límites entre lo público y lo privado fueran cada vez más difusos.

-Por ejemplo: un muchacho le confiesa a otra que le gusta ¿y la otra qué hace? Pantallazo, y lo publican. Entonces pues es más por publicidad, estar en riesgo constante de que todo el mundo se entere. 
-Muchas personas tienen acceso a información íntima que solo uno debería saber y no publicársela a todo el mundo, porque eso le puede jugar en contra a uno. No siempre puede ser bueno.

-Hay muchas relaciones que se han dañado por el Facebook, porque todo lo sabe todo mundo, aunque uno no crea. (G. 1, p. 16 , rr. 14,15 y 19)

La globalización resultó ser el tercer gran nodo del discurso de los estudiantes sobre el mundo actual. Este es un concepto sombrilla que los participantes utilizaron para referirse, por ejemplo, al capitalismo, a causas políticas de índole diversa, a hábitos de consumo, etc., desde una perspectiva inter y/o transnacional. Así pues, en torno a la globalización convergen y se agrupan todas las menciones a las dinámicas políticas y económicas que resultan significativas para los estudiantes. Tal vez por ello, la globalización significa, por un lado, tolerancia y apertura a otras culturas, mientras que, por otro, equivale a alienación, consumismo y exclusión social, tal y como se evidencia en este par de intervenciones:

-En un escenario globalizado, donde se ve disminuida la discriminación hacia grupos minoritarios, donde paso a paso se está viendo más normal esto, pues obviamente vamos a poder convivir mejor (...). Nosotros no podemos compararnos como antes que se veía a una persona de color en la calle y sencillamente si iba un blanco se tenía que cambiar de andén. Y lo bueno es que, hoy en día para nosotros, es absolutamente anormal y de hacerlo sería discriminatorio. Entonces, claro que se han venido cambiando las concepciones a raíz de la globalización, y se ha logrado, creo, una mejor convivencia entre diferentes culturas o grupos minoritarios. (G. 10, p. 5, r. 1)

-(...) yo digo que también influye lo de la globalización porque nosotros queremos adoptar unas culturas que no son propias de nosotros. Para el caso de Colombia, estamos adaptando culturas y costumbres, digamos, como comer hamburguesas (...) no es de necesidad, es más un deseo porque nosotros no tenemos suficiente con todo lo que tenemos. Con todo lo que produce 
Colombia, no tenemos necesidad de consumir comidas rápidas (...). Entonces pienso que es también adoptar esto de los países desarrollados. (G. 1, p. 15, r. 3)

A juicio de los participantes la globalización se experimenta de formas diferentes según el grado de desarrollo y producción tecnológica de cada sociedad. En consonancia con lo dicho en el numeral 3.1.3, la tecnología aparece de nuevo como un símbolo, en este caso del mundo globalizado. Según indicaron, mientras que en los países desarrollados se produce y se accede fácilmente a las tecnologías, en los países subdesarrollados las tecnologías se adoptan a un coste económico y social alto. Esto define el grado de influencia y el poder que ejercen los unos sobre los otros. Respecto a las disyuntivas entre primer/tercer mundo, o centro/periferia, o desarrollo/subdesarrollo ${ }^{13}$, etc., destacan afirmaciones como estas:

-Es como decir que nosotros somos los subdesarrollados y ellos los desarrollados, nosotros queremos llegar a ser los desarrollados y estamos adoptando culturas que para nosotros no son subdesarrolladas. (G. 1, p. 15, r. 3)

-Es que no las sabemos utilizar [las tecnologías] aquí en los países tercermundistas; llegamos tarde a todo por estar en eso; no hacemos las cosas por estar en eso; nos quedamos ahí y cuando nos damos cuenta se ha pasado el tiempo y hacemos nuestras tareas de forma rápida y mediocre. (G. 2, p. 5, r. 10)

\section{Debates en torno a la solidaridad, la identidad y el conocimiento}

Buena parte de las valoraciones más positivas sobre las tecnologías digitales salieron a la luz cuando se discutió sobre la cooperación y

13 Vale mencionar que de ello se da cuenta en la subsección 5.1.3, donde se argumenta que la tecnología se ubica en el centro del debate sobre la exclusión. 
la solidaridad en el mundo actual. Según los estudiantes, además de que las tecnologías digitales les permiten "estar más informados" (G. 10, p. 1, r. 7), también les facilitan el contacto y el trabajo a distancia, lo que redunda en una comunicación más fluida y en una difusión más veloz de la información. Por tanto, las distancias geográficas que antes imponían límites para la cooperación han dejado, a su juicio, de ser un obstáculo.

Los participantes afirmaron que a través de Internet, de las redes sociales y de los teléfonos móviles, etc., es posible adherirse a las causas que se consideran justas y ayudar a los demás bajo modalidades que hubieran resultado imposibles hasta hace poco. Según uno de ellos, "como [ahora] hay más contacto con las personas, se difunde la información. Hay más personas a favor de lo que se está haciendo; van a ayudarte y a colaborarte" (G. 9, p. 1, r. 3).

Para ilustrar sus argumentos, algunos participantes se refirieron al papel de las tecnologías digitales en los modos como ahora la gente coopera y se solidariza ante situaciones críticas de índole humanitaria o ambiental. Destacan estas frases, en las que se hace referencia a algunas de las problemáticas que para los estudiantes resultan más graves:

-(...) [La tecnología] también se puede usar para cosas buenas, positivas. Pues por ejemplo: lo que pasa en otros países, en Palestina ¿no? Uno se entera de lo que pasa en Palestina, de los abusos de los israelíes (...), entones uno da un apoyo o se puede sumar para uno tener un conocimiento de lo que pasa (...). Entonces si no sé, entonces me meto y busco y encuentro que hay personas de ese país que saben y le pueden decir a uno [lo que ocurre].

-Yo veo algo positivo con lo de las TIC: con lo del último desastre natural en Filipinas, (...) se organizaron conciertos que fueron transmitidos vía Internet, y la gente tenía la oportunidad de donar dinero nada más haciendo un clic y viendo el video. Creo que ese es un buen ejemplo de un buen uso de las TIC. (G. 10, p. 1, r. 4 y 8) 
-Sí, se coopera. Yo creo que uno a veces tiene una idea de que quiere hacer algo. Entonces cuando, por ejemplo, en Facebook, publican lo de la adopción de perritos, yo veo que la gente hace donaciones. Es que mucha gente no puede adoptar perritos porque no tiene los recursos para sostenerlos. Pero si algunos no colaboran para adoptar, entonces dicen "pues colaboremos con comida", o con plata para la esterilización también. (G. 9, p. 1, r. 8)

Por su parte, la discusión sobre la construcción identitaria tuvo un tomo mucho más polémico. Como se aprecia tanto en la Figura 2 como en buena parte de las intervenciones que se han reseñado hasta aquí, la construcción de la identidad es el tópico en el que concurren de forma más directa las posiciones de los estudiantes sobre la globalización, el acceso a la información y la comunicación mediada digitalmente.

La construcción identitaria parece ser una cuestión especialmente sensible para los jóvenes, pues saca a la luz el vínculo entre sus imaginarios sobre el mundo actual y el lugar que creen ocupar o que reclaman para sí. De sus intervenciones se deduce, de hecho, que reconocen la influencia que ejerce el mundo actual en las formas como se autoperciben y se proyectan ante los otros. Pareciera que para ellos la construcción identitaria es un proceso cada vez más reflexivo, dinámico y complejo. Esto debido a la variedad de fuentes informativas de que disponen, a los protocolos de la comunicación actual y a un mundo que perciben como económica y culturalmente interconectado.

Se habla enfáticamente de "construcción" identitaria, pues los participantes indicaron que, para ellos, la identidad no es algo estático e inmutable, sino que por el contrario es un proceso esencialmente dinámico. Los estudiantes insistieron en que viven en un mundo multicultural, en el que caben diversas opciones de vida y en el que las tecnologías digitales les permiten moldear su imagen a partir de sus intereses particulares. En sus propios términos: 
"cada vez tenemos más información, entonces tenemos más motivos para que seamos de cierta manera" (G. 2, p. 7, r. 1); "yo estoy detrás de una pantalla y puedo ser quien yo quiera, puedo buscar relacionarme con las personas que yo quiera" (G. 11, p. 3, r. 2).

Sin embargo, la reflexividad, dinamismo y complejidad que caracterizan la identidad en el mundo actual también tienen un lado problemático y riesgoso. Retorna, pues, la metáfora de la navaja del doble filo. Según indicaron, las tecnologías digitales permiten tal grado de control sobre la imagen propia que es muy fácil mostrar a los demás un retrato deliberadamente engañoso de sí mismo. Esto constituye uno de los problemas más graves de la comunicación digitalmente mediada, pues la manipulación malintencionada de la identidad merma la confianza y el sentido de la veracidad que dan soporte a las relaciones sociales significativas.

-(...) no es lo mismo ver a una persona en una pantalla a verla, a sentirla directamente (...) veo que ahora se crean vicios: la gente empieza a aparentar lo que no es. (G. 10, p. 4, r. 6)

-(...) en las redes sociales (...) hay como una doble faceta (...). No hay una comprensión de la realidad del sujeto y no hay un acercamiento a lo que es verdad. Hay una corrupción de la persona (...). Uno puede llegar a ser para la otra persona lo que uno quiera. (G. 11, p. 4, r. 4)

$\mathrm{Al}$ igual que como sucedió con la identidad, la discusión sobre la construcción de conocimiento estuvo fuertemente influenciada por las posiciones de los estudiantes respecto al acceso a la información y a la comunicación mediada digitalmente. En principio, se abordaron asuntos de índole informacional que dieron lugar a debates y cuestionamientos sobre la calidad y la veracidad de la información que está en la red. Esto les llevó, acto seguido, a referirse a una serie de mediaciones críticas que, según ellos, son imprescindibles para manejarse en un mundo donde la información 
es abundante y accesible. A su juicio, urge diferenciar entre facilidad, y calidad.

- $\mathrm{Si}$ (se) plantea que mejor es más rápido, sí [es mejor]; pero si se plantea que mejor es calidad, entonces no, porque lamentablemente eso es lo que se ha vivido mucho con las TIC e Internet: que una investigación o tarea la resolvemos muy rápido, en cuestión de un día. Pero la información que está ahí (...) solo la copias y ya no te esfuerzas por llegar más a fondo (...). Hay muchos aspectos que se perdieron, lastimosamente.

-Pues para (...) resolver problemas [Internet] sí ayuda: la facilidad, la rapidez del intercambio de información. Pero a la hora del punto clave como humanos, de razonar, no (...) no está ese sentido del debate, que podamos intercambiar opiniones, hacer críticas constructivas, cosas como esas que pueden aportar conocimiento sobre el tema; falta esa opinión personal. (G. 9, p. 5 , r. 1 y 5$)$

Algo parecido sucedió con la veracidad de la información, a la que se aludió en los siguientes términos:

-En internet nos encontramos con medios que no sabemos si son legítimos, que el filtro no es muy bueno porque cualquiera pude entrar, comentar lo que quiera, decir lo que quiera sin saber si es verídico. (G. 7, p. 6, r. 5)

-(...) El conocimiento lo tenemos al alcance de un clic, pero el problema es la fuente, qué tan confiable es. (G. 10, p. 2, r. 5)

$-(. .$.$) hay que ver realmente qué tan bueno es que puedas abrir$ una página web y creer todo lo que te está diciendo sin consultar una segunda o tercera fuente, porque no sabes si lo que te están diciendo es verdad o mentira y si el conocimiento que se está fabricando es falso, erróneo, que no tiene peso. (G. 10, p. 3, r. 2)

Los participantes insistieron bastante en que la información a la que se accede a través de Internet no está exenta de ser manipulada, por lo que es necesario "discernir y enfocarse" (G. 8, p. 4, r. 2) 
para evitar el desgaste que produce la cantidad de información que inunda la red. A su juicio, "hay que diferenciar entre buscar [información] y construir [conocimiento]" (G. 9, p. 2, r. 3) y es precisamente la falta de juicio crítico y de esfuerzo aquello que lo impide. A su juicio, vivimos en un mundo sospechosamente dócil y acrítico, en donde las personas se contentan con la información que les proveen los medios de comunicación y que aparece en Internet.

-Está más que demostrado que los medios de comunicación manipulan la información. Entonces, lo que hacen muchas personas es quedarse con eso. ¿Cuáles son las casusas y el porqué de las situaciones? No lo razonan (...) no buscan el porqué de las situaciones, se quedan con el punto de vista de los medios (...). Entonces creo que es algo muy mediático, las herramientas están, pero no las utilizan. (G. 9, p. 6, r. 1)

-No se aporta nada al conocimiento. Como decían: no se piensa, se busca la forma fácil. Entonces, no se busca como un estudio emancipatorio donde se vaya más allá, sino que nos quedamos con la primera o segunda fuente, pero ¿qué pasa más allá? Siempre nos quedamos con lo que está al alcance y no buscamos profundidad y no aportamos al desarrollo. (G. 10, p. 3, r. 3)

El esfuerzo y la capacidad de juicio aparecen, entonces, en el centro del debate sobre la construcción de conocimiento en el mundo actual. La importancia que les conceden los estudiantes es tanta que su carencia hace que se popularicen prácticas como el copy/paste, o que se confunda la argumentación crítica con la mera opinión personal.

-La facilidad de lo rápido nos vuelve perezosos. No vamos más allá sino solo copiar y pegar. (G. 8, p. 4, r. 4)

-Lastimosamente, la cultura del copy/paste hace que a veces el conocimiento y la construcción no se dé, sino que se estanquen los procesos $(. .$.$) la investigación y el conocimiento poco apa-$ recen. (G. 9, p. 2, r. 9) 
-Todas las personas tienen esa libertad de expresión dentro de una red y pueden decir lo que se les antoja y no necesitan un sustento científico, un sustento verídico de lo que están diciendo. Entonces, el conocimiento se vuelve algo superficial y banal y no es construcción de conocimiento, sino opinaderos. (G. 11, p. 2, r. 4)

-(...) la crítica no fue tomada como lo que es, sino como esa diversidad de opiniones sobre el mismo tema. Las personas a través de las TIC tienen la oportunidad para subir la información o no, pero no hay una herramienta crítica primordial o esencial que nos dé a nosotros un fundamento o una postura. (G. 11, p. 6, r. 5) 
